\title{
Influence and Passivity in Social Media
}

\author{
Daniel M. Romero \\ Cornell University \\ Center for Applied \\ Mathematics \\ Ithaca, New York, USA \\ dmr239@cornell.edu
}

\author{
Sitaram Asur \\ Social Computing Lab \\ HP Labs \\ Palo Alto, California, USA \\ sitaram.asur@hp.com
}

\author{
Wojciech Galuba \\ EPFL \\ Distributed Information \\ Systems Lab \\ Lausanne, Switzerland \\ wojciech.galuba@epfl.ch
}

\author{
Bernardo A. Huberman \\ Social Computing Lab \\ HP Labs \\ Palo Alto, California, USA \\ bernardo.huberman@hp.com
}

\begin{abstract}
The ever-increasing amount of information flowing through Social Media forces the members of these networks to compete for attention and influence by relying on other people to spread their message. A large study of information propagation within Twitter reveals that the majority of users act as passive information consumers and do not forward the content to the network. Therefore, in order for individuals to become influential they must not only obtain attention and thus be popular, but also overcome user passivity. We propose an algorithm that determines the influence and passivity of users based on their information forwarding activity. An evaluation performed with a 2.5 million user dataset shows that our influence measure is a good predictor of URL clicks, outperforming several other measures that do not explicitly take user passivity into account. We also explicitly demonstrate that high popularity does not necessarily imply high influence and vice-versa.
\end{abstract}

\section{INTRODUCTION}

The explosive growth of Social Media has provided millions of people the opportunity to create and share content on a scale barely imaginable a few years ago. Massive participation in these social networks is reflected in the countless number of opinions, news and product reviews that are constantly posted and discussed in social sites such as Facebook, Digg and Twitter, to name a few. Given this widespread generation and consumption of content, it is natural to target one's messages to highly connected people who will propagate them further in the social network. This is particularly the case in Twitter, which is one of the fastest growing social networks on the Internet, and thus the focus of advertis-

Permission to make digital or hard copies of all or part of this work for personal or classroom use is granted without fee provided that copies are not made or distributed for profit or commercial advantage and that copies bear this notice and the full citation on the first page. To copy otherwise, to republish, to post on servers or to redistribute to lists, requires prior specific permission and/or a fee.

Copyright 20XX ACM X-XXXXX-XX-X/XX/XX ...\$10.00. ing companies and celebrities eager to exploit this vast new medium. As a result, ideas, opinions, and products compete with all other content for the scarce attention of the user community. In spite of the seemingly chaotic fashion with which all these interactions take place, certain topics manage to get an inordinate amount of attention, thus bubbling to the top in terms of popularity and contributing to new trends and to the public agenda of the community. How this happens in a world where crowdsourcing dominates is still an unresolved problem, but there is considerable consensus on the fact that two aspects of information transmission seem to be important in determining which content receives inordinate amounts of attention.

One is the popularity and status of given members of these social networks, which is measured by the level of attention they receive in the form of followers who create links to their accounts to automatically receive the content they generate. The other is the influence that these individuals wield, which is determined by the actual propagation of their content through the network. This influence is determined by many factors, such as the novelty and resonance of their messages with those of their followers and the quality and frequency of the content they generate. Equally important is the passivity of members of the network which provides a barrier to propagation that is often hard to overcome. Thus gaining knowledge of the identity of influential and least passive people in a network can be extremely useful from the perspectives of viral marketing, propagating one's point of view, as well as setting which topics dominate the public agenda.

In this paper, we analyze the propagation of web links on twitter over time to understand how attention to given users and their influence is determined. We devise a general model for influence using the concept of passivity in a social network and develop an efficient algorithm similar to the HITS algorithm 14 to quantify the influence of all the users in the network. Our influence measure utilizes both the structural properties of the network as well as the diffusion behavior among users. The influence of a user thus depends on not only the size of the influenced audience, but also on their passivity. This differentiates it from earlier measures of influence which were primarily based on individual statistical properties such as the number of followers or retweets 7 . 
We have shown through our extensive evaluation that this influence model outperforms other measures of influence such as PageRank, H-index, the number of followers and the number of retweets. In addition it has good predictive properties in that it can forecast in advance the upper bound on the number of clicks a URL can get. We have also presented case studies showing the top influential users uncovered by our algorithm. An important conclusion from the results is that the correlation between popularity and influence is quite weak, with the most influential users not necessarily the ones with the highest popularity. Additionally, when we considered nodes with high passivity, we found a majority of them to be spammers and robot users. This demonstrates an application of our algorithm in automatic user categorization and filtering of online content .

\section{RELATED WORK}

The study of information and influence propagation in social networks has been particularly active for a number of years in fields as disparate as sociology, communication, marketing, political science and physics. Earlier work focused on the effects that scale-free networks and the affinity of their members for certain topics had on the propagation of information 6. Others discussed the presence of key influentials $12,11,8,5,10$ in a social network, defined as those who are responsible for the overall information dissemination in the network. This research highlighted the value of highly connected individuals as key elements in the propagation of information through the network.

Huberman et al. 2] studied the social interactions on Twitter to reveal that the driving process for usage is a sparse hidden network underlying the friends and followers, while most of the links represent meaningless interactions. Jansen et al. 3] have examined twitter as a mechanism for word-of-mouth advertising. They considered particular brands and products and examined the structure of the postings and the change in sentiments. Galuba et al. 4 . propose a propagation model that predicts, which users will tweet about which URL based on the history of past user activity.

There have also been earlier studies focused on social influence and propagation. Agarwal et al. 8 have examined the problem of identifying influential bloggers in the blogosphere. They discovered that the most influential bloggers were not necessarily the most active. Aral et al 9] have distinguished the effects of homophily from influence as motivators for propagation. As to the study of influence within twitter, Cha et al. 7] have performed a comparison of three different measures of influence - indegree, retweets and user mentions. They discovered that while retweets and mentions correlated well with each other, the indegree of users did not correlate well with the other two measures. Based on this, they hypothesized that the number of followers may not a good measure of influence. On the other hand, Weng et al [5] have proposed a topic-sensitive PageRank measure for influence in Twitter. Their measure is based on the fact that they observed high reciprocity among follower relationships in their dataset, which they attributed to homophily. However, other work 7. has shown that the reciprocity is low overall in Twitter and contradicted the assumptions of this work.

\section{TWITTER}

\subsection{Background on Twitter}

Twitter is an extremely popular online microblogging service, that has gained a very large user base, consisting of more than 105 million users (as of April 2010). The Twitter graph is a directed social network, where each user chooses to follow certain other users. Each user submits periodic status updates, known as tweets, that consist of short messages limited in size to 140 characters. These updates typically consist of personal information about the users, news or links to content such as images, video and articles. The posts made by a user are automatically displayed on the user's profile page, as well as shown to his followers.

A retweet is a post originally made by one user that is forwarded by another user. Retweets are useful for propagating interesting posts and links through the Twitter community.

Twitter has attracted lots of attention from corporations for the immense potential it provides for viral marketing. Due to its huge reach, Twitter is increasingly used by news organizations to disseminate news updates, which are then filtered and commented on by the Twitter community. A number of businesses and organizations are using Twitter or similar micro-blogging services to advertise products and disseminate information to stockholders.

\subsection{Dataset}

Twitter provides a Search API for extracting tweets containing particular keywords. To obtain the dataset for this study, we continuously queried the Twitter Search API for a period of 300 hours starting on 10 Sep 2009 for all tweets containing the string http. This allowed us to acquire a continuous stream of 22 million tweets with URLs, which we estimated to be $1 / 15$ th of the entire Twitter activity at that time. From each of the accumulated tweets, we extracted the URL mentions. Each of the unique 15 million URLs in the data set was then checked for valid formatting and the URLs shortened via the services such as bit.ly or tinyurl.com were expanded into their original form by following the HTTP redirects. For each encountered unique user ID, we queried the Twitter API for metadata about that user and in particular the user's followers and followees. The end result was a dataset of timestamped URL mentions together with the complete social graph for the users concerned.

User graph. The user graph contains those users whose tweets appeared in the stream, i.e., users that during the 300 hour observation period posted at least one public tweet containing a URL. The graph does not contain any users who do not mention any URLs in their tweets or users that have chosen to make their Twitter stream private.

For each newly encountered user ID, the list of followed users was only fetched once. Our data set does not capture the changes occurring in the user graph over the observation period.

\section{THE IP ALGORITHM}

Evidence for passivity. The users that receive information from other users may never see it or choose to ignore it. We have quantified the degree to which this occurs on Twitter (Fig. 4). An average Twitter user retweets only one in 318 URLs, which is a relatively low value. The retweeting rates vary widely across the users and the small number of 


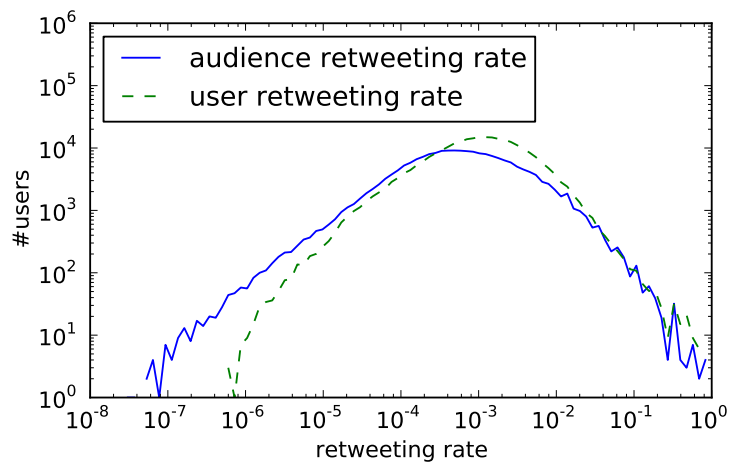

Figure 1: Evidence for the Twitter user passivity. We measure passivity by two metrics: 1 . the user retweeting rate and 2 . the audience retweeting rate. The user retweeting rate is the ratio between the number of URLs that user $i$ decides to retweet to the total number of URLs user $i$ received from the followed users. The audience retweeting rate is the ratio between the number of user $i$ 's URLs that were retweeted by $i$ 's followers to the number of times a follower of $i$ received a URL from $i$.

the most active users play an important role in spreading the information in Twitter. This suggests that the level of user passivity should be taken into account for the information spread models to be accurate.

Assumptions. Twitter is used by many people as a tool for spreading their ideas, knowledge, or opinions to others. An interesting and important question is whether it is possible to identify those users who are very good at spreading their content, not only to those who choose to follow them, but to a larger part of the network. It is often fairly easy to obtain information about the pairwise influence relationships between users. In Twitter, for example, one can measure how much influence user A has on user B by counting the number of times B retweeted A. However, it is not very clear how to use the pairwise influence information to accurately obtain information about the relative influence each user has on the whole network. To answer this question we design an algorithm (IP) that assigns a relative influence score and a passivity score to every user. The passivity of a user is a measure of how difficult it is for other users to influence him. We assume that the influence of a user depends on both the quantity and the quality of the audience she influences. In general, our model makes the following assumptions:

1. A user's influence score depends on the number of people she influences as well as their passivity.

2. A user's influence score depends on how dedicated the people she influences are. Dedication is measured by the amount of attention a user pays to a given one as compared to everyone else.

3. A user's passivity score depends on the influence of those who she's exposed to but not influenced by.

4. A user's passivity score depends on how much she rejects other user's influence compared to everyone else.

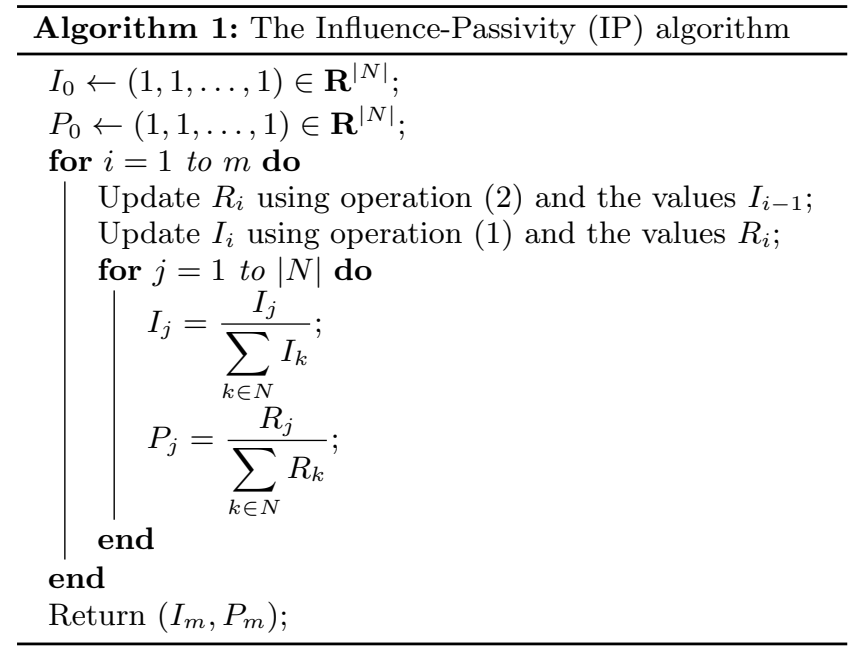

Operation. The algorithm iteratively computes both the passivity and influence scores simultaneously in a similar manner as in the HITS algorithm for finding authoritative web pages and hubs that link to them 14 .

Given a weighted directed graph $G=(N, E, W)$ with nodes $N$, arcs $E$, and arc weights $W$, where the weights $w_{i j}$ on arc $e=(i, j)$ represent the ratio of influence that $i$ exerts on $j$ to the total influence that $i$ attempted to exert on $j$, the IP algorithm outputs a function $I: N \rightarrow[0,1]$, which represents the nodes' relative influence on the network, and a function $P: N \rightarrow[0,1]$ which represents the nodes' relative passivity of the network.

For every arc $e=(i, j) \in E$, we define the acceptance rate by $u_{i j}=\frac{w_{i, j}}{\sum_{k:(k, j) \in E} w_{k j}}$. This value represents the amount of influence that user $j$ accepted from user $i$ normalized by the total influence accepted by $j$ from all users on the network. The acceptance rate can be viewed as the dedication or loyalty user $j$ has to user $i$. On the other hand, for every $e=(j, i) \in E$ we define the rejection rate by $v_{j i}=\frac{1-w_{j i}}{\sum_{k:(j, k) \in E}\left(1-w_{j k}\right)}$. Since the value $1-w_{j i}$ is amount of influence that user $i$ rejected from $j$, then the value $v_{j i}$ represents the influence that user $i$ rejected from user $j$ normalized by the total influence rejected from $j$ by all users in the network.

The algorithm is based on the following operations:

$$
\begin{aligned}
I_{i} & \leftarrow \sum_{j:(i, j) \in E} u_{i j} P_{j} \\
P_{i} & \leftarrow \sum_{j:(j, i) \in E} v_{j i} I_{j}
\end{aligned}
$$

Each term on the right hand side of the above operations corresponds to one of the listed assumptions. In operation 1 the term $P_{j}$ corresponds to assumption 1 and the term $u_{i j}$ corresponds to assumption 2 . In operation 2 the term $I_{j}$ corresponds to assumption 3 and the term $v_{j i}$ corresponds to assumption 4. The Influence-Passivity algorithm (Algorithm 1) takes the graph $G$ as the input and computes the influence and passivity for each node in $m$ iterations. 
Generating the input graph. There are many ways of defining the influence graph $G=(N, E, W)$. We choose to construct it by taking into account retweets and the follower graph in the following way: The nodes are users who tweeted at least 3 URLs. The arc $(i, j)$ exists if user $j$ retweeted a URL posted by user $i$ at least once. The arc $e=(i, j)$ has weight $w_{e}=\frac{S_{i j}}{Q_{i j}}$ where $Q_{i}$ is the number of URLs that $i$ mentioned and $S_{i j}$ is the number of URLs mentioned by $i$ and retweeted by $j$.

\section{EVALUATION}

\subsection{Computations}

Based on the obtained dataset $(3.2)$ we generate the weighted graph using the method described in 4 The graph consists of approximately 450k nodes and 1 million arcs with mean weight of 0.07 , and we use it to compute the PageRank, influence and passivity values for each node. The Influence-Passivity algorithm (Algorithm converges to the final values in tens of iterations (Fig. 2).

PageRank. The PageRank algorithm has been widely used to rank web pages as well as people based on their authority and their influence 13.5. In order to compare it with the results from the IP algorithm, we compute PageRank on the weighted graph $G=(N, E, W)$ with a small change. First, since the $\operatorname{arcs} e=(i, j) \in E$ indicate that user $i$ exerts some influence on user $j$ then we invert all the arcs before running PageRank on the graph while leaving the weights intact. In other words, we generate a new graph $G^{\prime}=\left(N^{\prime}, E^{\prime}, W^{\prime}\right)$ where $N^{\prime}=N, E^{\prime}=\{(i, j):(j, i) \in E\}$, and for each $(i, j) \in E^{\prime}$ we define $w_{i j}^{\prime}=w_{j i}$. This generates a new graph $G^{\prime}$ analogous to $G$ but where the influenced users point to their influencers. Second, since the graph $G^{\prime}$ is weighted we assume that when the the random surfer of the PageRank algorithm is currently at the node $i$, she chooses to visit node $j$ next with probability $\frac{w_{i j}^{\prime}}{\sum_{k:(i, k) \in E^{\prime}} w_{i k}^{\prime}}$.

The Hirsch Index. The Hirsch index (or H-index) is used in the scientific community in order to measure the productivity and impact of a scientist. A scientist has index $h$ if he has published $h$ articles which have been cited at least $h$ times each. It has been shown that the H-index is a good indicator of whether a scientist has had high achievements such as getting the Nobel prize 16. Analogously, in Twitter, a user has index $h$ if $h$ of his URL posts have been retweeted at least $h$ times each.

\subsection{Influence as a correlate of attention}

Any measure of influence is necessarily a subjective one. However, in this case, a good measure of influence should have a high predictive power on how well the URLs mentioned by the influential users attract attention and propagate in the social network. We would expect the URLs that highly influential users propagate to attract a lot of attention and user clicks. Thus, a viable estimator of attention is the number of times a URL has been accessed.

Click data. Bit.ly is a URL shortening service that for each shortened URL keeps track of how many times it has been accessed. For the 3.2M Bit.ly URLs found in the tweets we have queried the Bit.ly API for the number of clicks the service has registered on that URL.

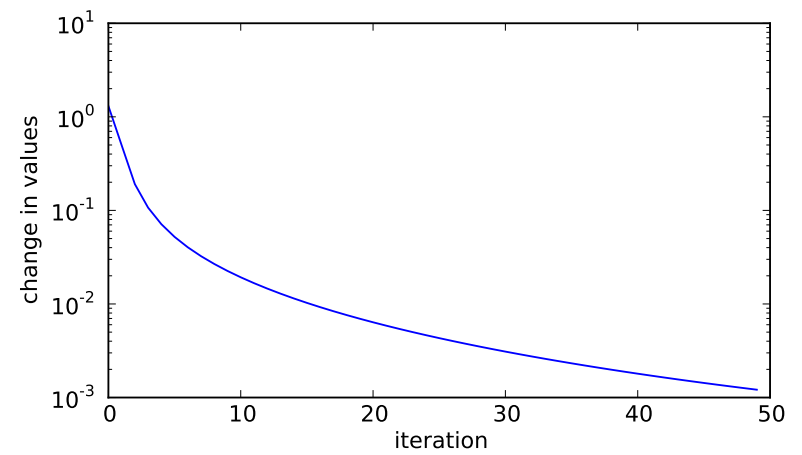

Figure 2: IP-algorithm convergence. In each iteration we measure the sum of all the absolute changes of the computed influence and passivity values since the previous iteration

URL traffic correlation. Using the URL click data, we take several different user attributes and test how well they can predict the attention the URLs posted by the users receive (Fig. 3). It is important to note that none of the influence measures are capable of predicting the exact number of clicks. The main reason for this is that the amount of attention a URL gets is not only a function of the influence of the users mentioning it, but also of many other factors including the virality of the URL itself and more importantly, whether the URL was mentioned anywhere outside of Twitter, which is likely to be the biggest source of unpredictability in the click data. The click data that we collected represents the total clicks on the URLs.

The wide range of factors potentially affecting the Bit.ly clicks may prevent us from predicting their number accurately. However, the upper bound on that number can to a large degree be predicted. To eliminate the outlier cases, we examined how the 99.9th percentile of the clicks varied as the measure of influence increased.

Number of followers. The most readily available and often used by the Twitterers measure of influence is the number of followers a user has. As the Figure 3(a) shows, the number of followers of an average poster of a given URL is a relatively weak predictor of the maximum number of clicks that the URL can receive.

Number of retweets. When users post URLs their posts might be retweeted by other users. Each retweet explicitly credits the original poster of the URL (or the user from whom the retweeting user heard about the URL). The number of times a user has been credited in a retweet has been assumed to be a good measure of influence 77. However, Figure 3(b) shows that the number of times a user has been retweeted in the past is a poor predictor of the maximum number of clicks the URLs posted by that user can get.

PageRank. Figure 3(c) shows that the average PageRank of those who tweet a certain URL does not correlate well with the number of clicks the URL will get. One of the main differences between the IP algorithm and PageRank is that the IP algorithm takes into account the passivity of the people a user influences and PageRank does not.IP-influence 


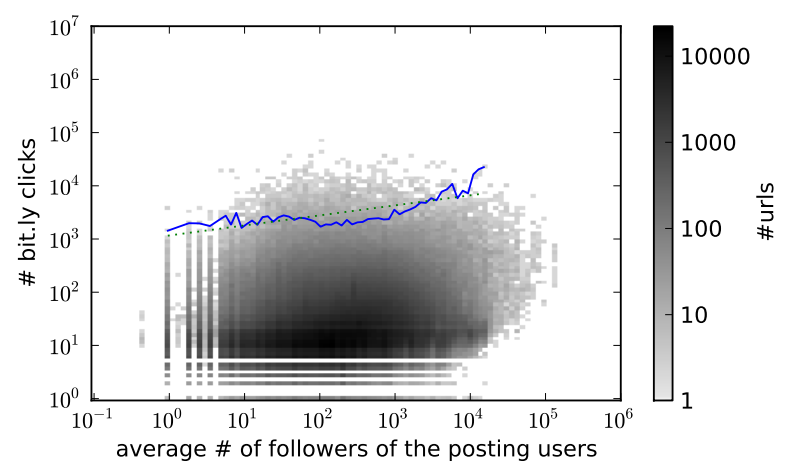

(a) Average number of followers vs. number of clicks on URLs

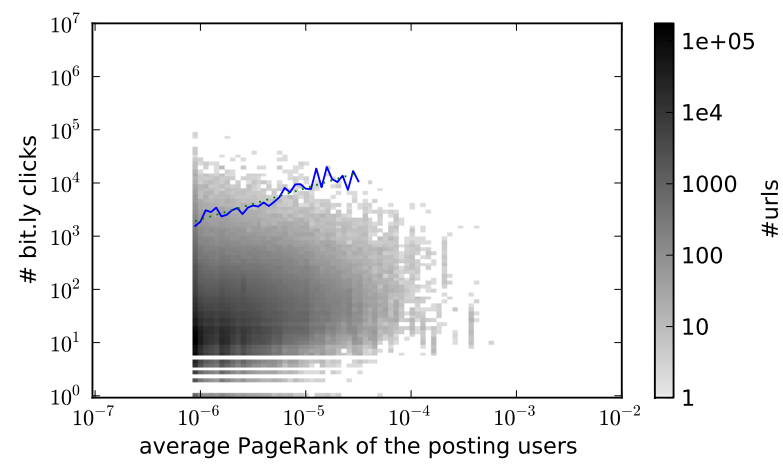

(c) Average user PageRank vs. number of clicks on URLs

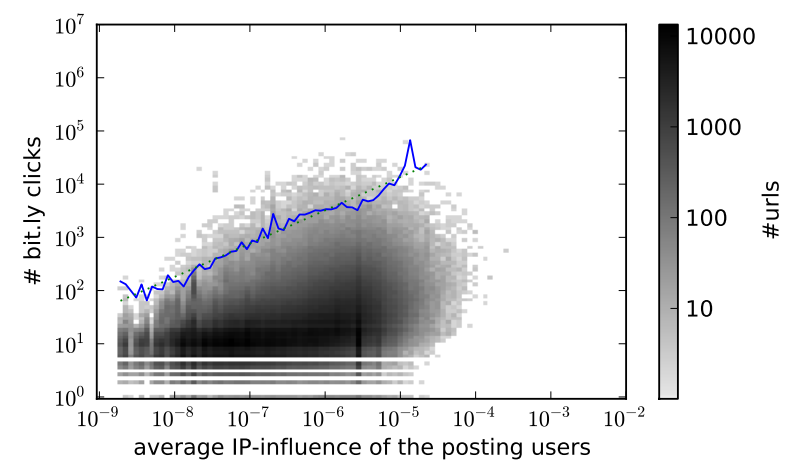

(e) Average user IP-influence vs. number of clicks on URLs, using the retweet graph as input

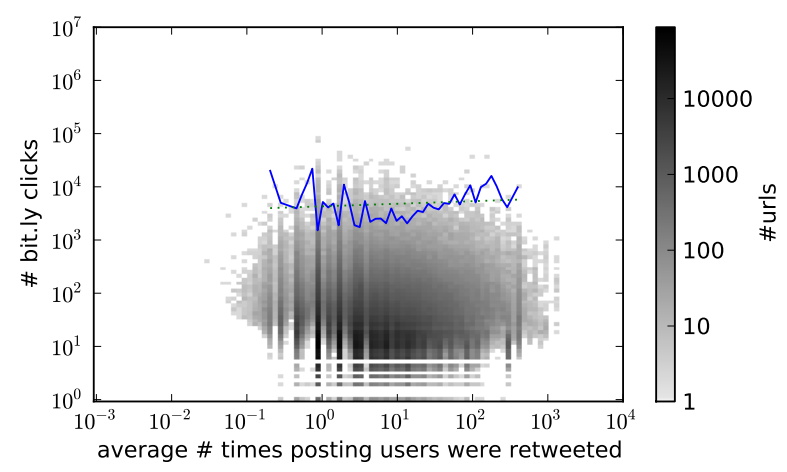

(b) Average number of times users were retweeted vs. number of clicks on URLs

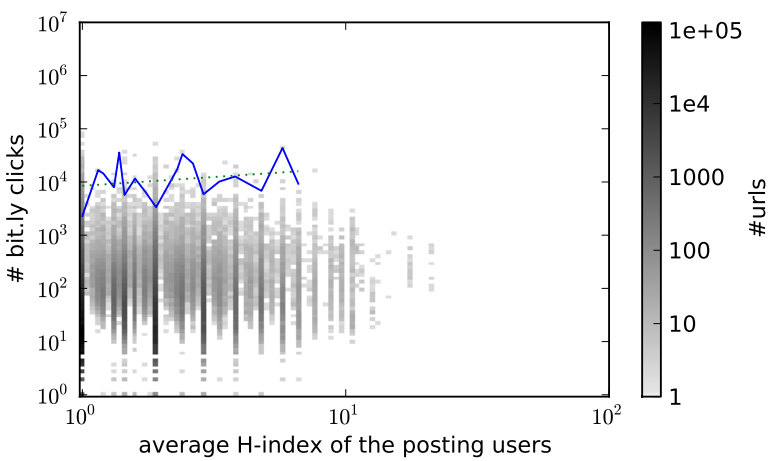

(d) Average user H-index vs. number of clicks on URLs

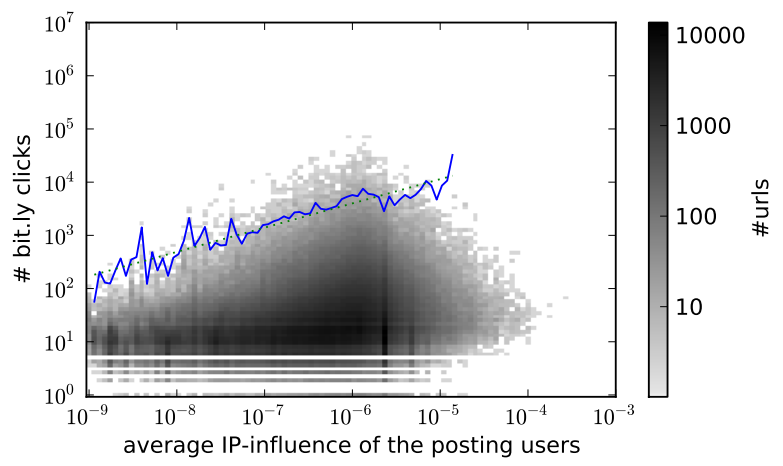

(f) Average user IP-influence vs. number of clicks on URLs, using the co-mention graph as input

Figure 3: We consider several user attributes: the number of followers, the number of times a user has been retweeted, the user's PageRank, H-index and IP-influence. For each of the 3.2M Bit.ly URLs we compute the average value of a user's attribute among all the users that mentioned that URL. This value becomes the $x$ coordinate of the URL-point; the $y$ coordinate is the number of clicks on the Bit.ly URL. The density of the URL-points is then plotted for each of the four user attributes. The solid line in each figure represents the 99.9th percentile of Bit.ly clicks at a given attribute value. The dotted line is the linear regression fit for the solid line. 


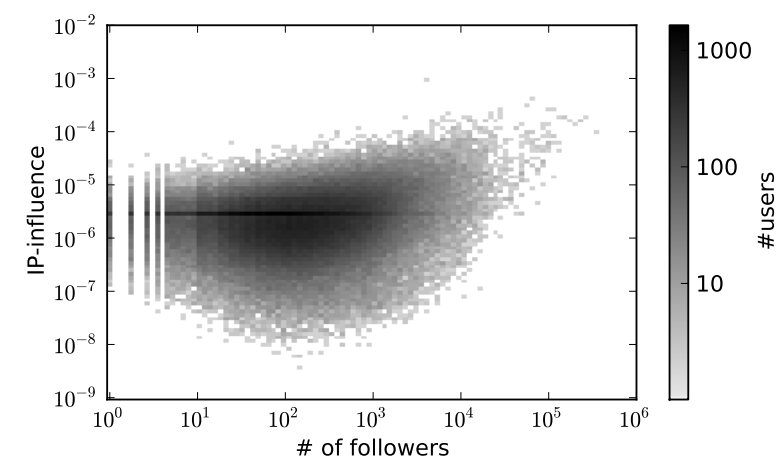

Figure 4: For each user we place a user-point with IP-influence as the $y$ coordinate and the $x$ coordinate set to the number of user's followers. The density of user-points is represented in grayscale.

is a much better indicator of URL popularity than PageRank. This suggests that influencing users who are difficult to influence, as opposed to simply influencing many users, has a positive impact on the eventual popularity of the message that a user tweets.

The Hirsch Index. Figure 3(d) shows that despite the fact that in the scientific community the H-index is used as a good predictor of high achievements, in Twitter it does not correlate well with URL popularity. This may reflect the fact that attention in the scientific community plays a symmetric role, since those who pay attention to the work of others also seek it from the same community. Thus, citations play a strategic role in the successful publishing of papers, since the expectation of authors is that referees and authors will demand attention to their work and those of their colleagues. Within Social Media such symmetry does not exist and thus the decision to forward a message to the network lacks this particularly strategic value.

IP-Influence score. As we can see in Figure 3(e) the average IP-influence of those who tweeted a certain URL can determine the maximum number of clicks that a URL will get. Since the URL clicks are never considered by the IP algorithm to compute the user's influence, the fact that we find a very clear connection between average IP-influence and the eventual popularity of the URLs (measured by clicks) serves as an unbiased evaluation of the algorithm and exposes the power of IP-influence. For example, as we can see in Figure 3(e) given a group of users having very large average IP-influence scores who post a URL we can estimate, with $99.9 \%$ certainty, that this URL will not receive more than 100, 000 clicks. On the other hand, if a group of users with very low average IP-influence score post the same URL we can estimate, with $99.9 \%$ certainty that the URL will not receive more than 100 clicks.

Furthermore, figure 4 shows that a user's IP-influence is not well correlated with the number of followers she has. This reveals interesting implications about the relationship between a person's popularity and the influence she has on other people. In particular, it shows that having many followers on Twitter does not imply power to influence them to even click on a URL.

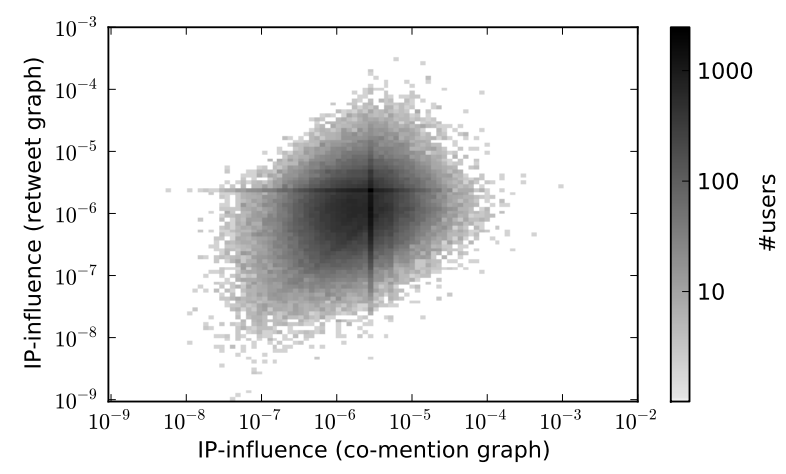

Figure 5: The correlation between the IP-influence values computed based on two inputs: the comention influence graph and the retweet influence graph.

\section{IP ALGORITHM ADAPTABILITY}

As mentioned earlier (4) there are many ways of defining a social graph in which the edges indicate pairwise influence. We have so far been using the graph based on which user retweeted which user (retweet influence graph). However, that explicitly signals of influence such as retweets are not always available. One way of overcoming this obstacle is to use other, possibly weaker, signals of influence. In the case of Twitter, we can define an influence graph based on mentions of URLs without regard of actual retweeting in the following way.

The co-mention graph. The nodes of the co-mention influence graph are users who tweeted at least three URLs. The edge $(i, j)$ exists if user $j$ follows user $i$ and $j$ mentioned at least one URL that $i$ had previously mentioned. The edge $e=(i, j)$ has weight $w_{e}=\frac{S_{i j}}{F_{i j}+S_{i j}}$ where $F_{i j}$ is the number of URLs that $i$ mentioned and $j$ never $\operatorname{did}$ and $S$ is the number of URLs mentioned by $j$ and previously mentioned by $i$.

The resulting graph has the disadvantage that the edges are based on a much less explicit notion of influence than when based on retweets. Therefore the graph could have edges between users who do not influence each other. On the other hand, the retweeting conventions on Twitter are not uniform and therefore sometimes users who repost a URL do not necessarily credit the correct source of the URL with a retweet 15. Hence, the influence graph based on retweets has potentially missing edges.

Since the IP algorithm has the flexibility of allowing any influence graph as input, we can compute the influence scores of the users based on the co-mention influence graph and compare with the results obtained from the retweet influence graph. As we can see in Figure 3(f) we find that the retweet graph yields influence scores that are better at predicting the maximum number of clicks a URL will obtain than the comention influence graph. Nevertheless, Figures 3(f), 3(c) $3(\mathrm{a})$ and 3(b) show that the influence values obtained from the co-mention influence graph are still better at predicting URL traffic than other measures such as PageRank, number of followers, H-index or the total number of times a user has been retweeted. Furthermore, Figure 5 shows that the 


\begin{tabular}{|l|l|}
\hline mashable & Social Media Blogger \\
jokoanwar & Film Director \\
google & Google News \\
aplusk & Actor \\
syfy & Science Fiction Channel \\
smashingmag & Online Developer Magazine \\
michellemalkin & Conservative Commentator \\
theonion & News Satire Organization \\
rww & Tech/Social Media Blogger \\
breakingnews & News Aggregator \\
\hline
\end{tabular}

Table 1: Users with the most IP-influence (with at least 10 URLs posted in the period)

influence score based on both graphs do not correlate well, which suggests that considering explicit vs. implicit signals of influence can drastically change the outcome of the IP algorithm. In general, we find that the explicitness of the signal provided by the retweets yields slightly better results when it comes to predicting URL traffic, however, the influence scores based on co-mentions may surface a different set of potentially influential users.

\section{CASE STUDIES}

As we mentioned earlier, one important application of the IP algorithm is ranking users by their relative influence. In this section, we present a series of rankings of Twitter users based on the influence, passivity, and number of followers.

The most influential. Table 1 shows the users with the most IP-influence in the network. We constrain the number of URLs posted to 10 to obtain this list, which is dominated by news services from politics, technology, and Social Media. These users post many links which are forwarded by other users, causing their influence to be high.

The most passive. Table 2 shows the users with the most IP-passivity in the network. Passive users are those who follow many people, but retweet a very small percentage of the information they consume. Interestingly, robot accounts (which automatically aggregate keywords or specific content from any user on the network), suspended accounts (which are likely to be spammers), and users who post extremely often are among the users with the most IP-passivity. Since robots "attend" to all existing tweets and only retweet certain ones, the percentage of information they forward from other users is actually very small. This explains why the IP-algorithm assigns them such high passivity scores. This also highlights a new application of the IP-algorithm: automatic identification of robot users including aggregators and spammers.

The least influential with many followers. We have demonstrated that the amount of attention a person gets may not be a good indicator of the influence they have in spreading their message. In order to make this point more explicit, we show, in Table 3, some examples of users who are followed by many people but have relatively low influence. These users are very popular and have the attention of millions of people but are not able to spread their message very far. In most cases, their messages are consumed by their followers but not considered important enough to forward to others.

The most influential with few followers. We are

\begin{tabular}{|l|l|}
\hline redscarebot & Keyword Aggregator \\
drunk_bot & Suspended \\
tea_robot & Keyword Aggregator \\
condos & Listing Aggregator \\
wootboot & Suspended \\
raybeckerman & Attorney \\
hashphotography & Keyword Aggregator \\
charlieandsandy & Suspended \\
ms_defy & Suspended \\
rpattinsonbot & Keyword Aggregator \\
\hline
\end{tabular}

Table 2: Users with the most IP-passivity

also able identify users with very low number of followers but high influence. Table 4 shows the users with the most influence who rank less that 100,000 in number of followers. We find that during the data collection period some of the users in this category ran very successful retweeting contests where users who retweeted their URLs would have the chance of winning a prize. Moreover, there is a group of users who post from Twitdraw.com, a website where people can make drawings and post them on Twitter. Even though these users don't have many followers, their drawings are of very high quality and spread throughout Twitter reaching many people. Other interesting users such as local politicians and political cartoonists are also found in the list. The IP-influence measure allows us to find interesting content posted by users who would otherwise be buried by popularity rankings such as number of followers.

\section{DISCUSSION}

Influence as predictor of attention. As we demonstrated in \$5, the IP-influence of the users is an accurate predictor of the upper bound on the total number of clicks they can get on the URLs they post. The input to the influence algorithm is a weighted graph, where the arc weights represent the influence of one user over another. This graph can be derived from the user activity in many ways, even in cases where explicit feedback in the form of retweets or "likes" is not available (\$6).

Topic-based and group-based influence. The InfluencePassivity algorithm can be run on a subpgraph of the full graph or on the subset of the user activity data. For example, if only users tweeting about a certain topic are part of the graph, the IP-influence determines the most influential users in that topic. It is an open question whether the IP algorithm would be equally accurate at different graph scales.

Content ranking. The predictive power of IP-influence can be used for content filtering and ranking in order to reveal content that is most likely to receive attention based on which users mentioned that content early on. Similarly, as in the case of users, this can be computed on a per-topic or per-user-group basis.

Content filtering. We have observed from our passivity experiments that highly passive users tend to be primarily robots or spammers. This leads to an interesting extension of this work to perform content filtering, limiting the tweets to influential users and thereby reducing spam in Twitter feeds.

Influence dynamics. We have computed the influence 


\begin{tabular}{|l|l|c|c|}
\hline User name & Category & Rank by \# followers & Rank by IP-influence \\
\hline thatkevinsmith & Screen Writer & 33 & 1000 \\
nprpolitics & Political News & 41 & 525 \\
eonline & TV Channel & 42 & 1008 \\
marthastewart & Television Host & 43 & 1169 \\
nba & Sports & 64 & 1041 \\
davidgregory & Journalist & 106 & 3630 \\
nfl & Sports & 110 & 2244 \\
cbsnews & News Channel & 114 & 2278 \\
jdickerson & Journalist & 148 & 4408 \\
newsweek & News Magazine & 148 & 756 \\
\hline
\end{tabular}

Table 3: Users with many followers and low relative influence

\begin{tabular}{|l|l|c|c|}
\hline User name & Category & Rank by \# followers & Rank by IP-influence \\
\hline cashcycle & Retweet Contest & 153286 & 13 \\
mobiliens & Retweet Contest & 293455 & 70 \\
jadermattos & Twitdraw & 227934 & 134 \\
_jaum_ & Twitdraw & 404385 & 143 \\
robmillerusmc & Congressional Candidate & 147803 & 145 \\
sitekulite & Twitdraw & 423917 & 149 \\
jesse_sublett & Musician & 385265 & 151 \\
cyberaurora & Tech News Website & 446207 & 163 \\
viveraxo & Twitdraw & 458279 & 165 \\
fireflower_- & Political Cartoons & 452832 & 195 \\
\hline
\end{tabular}

Table 4: Users with very few followers but high relative influence

measures over a fixed 300-hour window. However, the Social Media are a rapidly changing, real-time communication platform. There are several implications of this. First, the IP algorithm would need to be modified to take into account the tweet timestamps. Second, the IP-influence itself changes over time, which brings a number of interesting questions about the dynamics of influence and attention. In particular, whether users with spikes of IP-influence are overall more influential than users which can sustain their IP-influence over time is an open question.

\section{CONCLUSION}

Given the mushrooming popularity of Social Media, vast efforts are devoted by individuals, governments and enterprises to getting attention to their ideas, policies, products, and commentary through social networks. But the very large scale of the networks underlying Social Media makes it hard for any of these topics to get enough attention in order to rise to the most trending ones. Given this constraint, there has been a natural shift on the part of the content generators towards targeting those individuals that are perceived as influential because of their large number of followers. This study shows that the correlation between popularity and influence is weaker than it might be expected. This is a reflection of the fact that for information to propagate in a network, individuals need to forward it to the other members, thus having to actively engage rather than passively read it and cease to act on it. Moreover, since our measure of influence is not specific to Twitter it is applicable to many other social networks. This opens the possibility of discovering influential individuals within a network which can on average have a further reach than others in the same medium, regardless of their popularity.

\section{REFERENCES}

[1] Jure Leskovec, Lada A. Adamic and Bernardo A. Huberman. The dynamics of viral marketing. In Proceedings of the 7th ACM Conference on Electronic Commerce, 2006.

[2] Bernardo A. Huberman, Daniel M. Romero, and Fang Wu. Social networks that matter: Twitter under the microscope. First Monday, 14(1), Jan 2009.

[3] B. Jansen, M. Zhang, K. Sobel, and A. Chowdury. Twitter power: Tweets as electronic word of mouth. Journal of the American Society for Information Science and Technology, 2009.

[4] Wojciech Galuba, Karl Aberer, Dipanjan Chakraborty, Zoran Despotovic, Wolfgang Kellerer Outtweeting the Twitterers - Predicting Information Cascades in Microblogs 3rd Workshop on Online Social Networks, WOSN, 2010)

[5] Jianshu Weng and Ee-Peng Lim and Jing Jiang and Qi He. TwitterRank: finding topic-sensitive influential twitterers. WSDM, 2010.

[6] Fang Wu, Bernardo A. Huberman, Lada Adamic and Josh Tyler. Information Flow in Social Groups. Physica A, Vol 337, 327-335, 2004.

[7] Meeyoung Cha and Hamed Haddadi and Fabricio Benevenuto and Krishna P. Gummadi. Measuring User Influence in Twitter: The Million Follower Fallacy. 4th International AAAI Conference on Weblogs and Social Media (ICWSM), 2010.

[8] Nitin Agarwal and Huan Liu and Lei Tang and Philip $\mathrm{S}$. Yu. Identifying the Influential Bloggers in a 
Community. WSDM, 2008.

[9] Sinan Aral, Lev Muchnik and Arun Sundararajan.

Distinguishing influence-based contagion from

homophily-driven diffusion in dynamic networks.

Proceedings of the National Academy of Sciences, Vol. 106 (51), pp. 21544-21549, 2009.

[10] Duncan J. Watts and Peter Sheridan Dodds. Influentials, Networks, and Public Opinion Formation. Journal of Consumer Research, Vol. 34 (4), pp. 441-458, 2007.

[11] Amit Goyal, Francesco Bonchi and Laks V.S. Lakshmanan. Learning Influence Probabilities In Social Networks. WSDM, 2010.

[12] P. Domingos and M. Richardson. Mining the network value of customers. SIGKDD, 2001.

[13] S. Brin and L. Page. The anatomy of a large-scale hypertextual Web search engine. Computer Networks and ISDN Systems, Vol 30, 1-7, 1998.

[14] Jon Kleinberg. Authoritative sources in a hyperlinked environment. Journal of the ACM 46 (5): 604 -632, 1999.

[15] Boyd Danah, Scott Golder, and Gilad Lotan. Tweet, Tweet, Retweet: Conversational Aspects of Retweeting on Twitter. HICSS-43. IEEE 2010.

[16] Jorge E. Hirsch. An index to quantify an individual's scientific research output. Proceedings of the National Academy of Sciences 102(46): 16569 -16572, 2005. 